\title{
Some Unresolved Problems in Magnetism
}

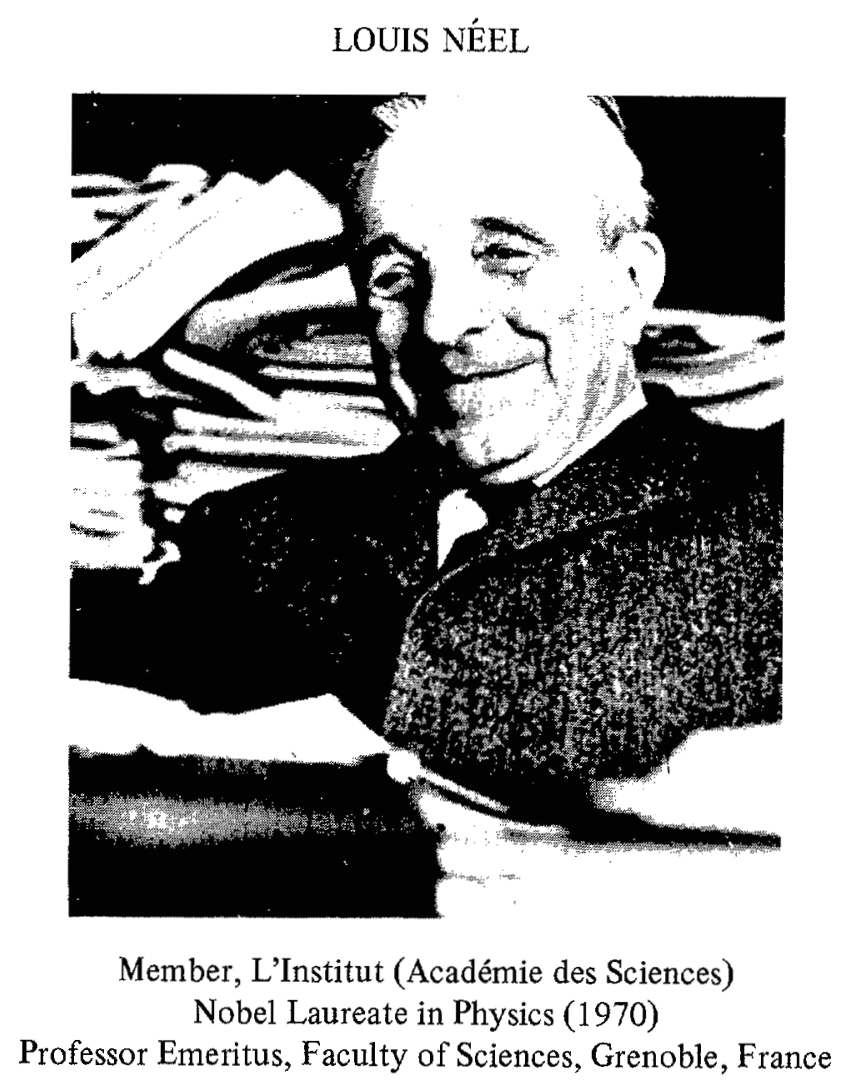

$I^{1}$

T IS with much pleasure that I stand here today amidst such a numerous congregation of specialists in magnetism and its applications. The outlook of this branch of physics has changed a lot since the early years, more than 55 years ago, when I happened to get involved with it. If my memory does not betray me, that was in the cellars of the Ecole Normale Supérieure, where my friend Bernamont was repeating Sixtus and Tonks' very recent experiments on the propagation of Barkhausen discontinuities, which are unfairly forgotten nowadays. Three years later in Strasburg, I joined the laboratory created by Pierre Weiss, after managing a very active magnetism center in Zürich. It is always an instructive thing to place oneself within the atmosphere of that period, although those days were prehistorical as compared to contemporary science, when a tenyear-old reference is already an old one.

At that time, though, the experimental knowledge of the magnetic properties of solids was fairly well-developed. It was already an old science, since the strange properties of magnetite or lodestone had attracted some attention for nearly 3000 years. On the other hand, theoretical knowledge remained rather embryonic. Only Langevin's theory of diamagnetism has stood almost untouched by time until now, whereas his theory of paramagnetism, classical in its development, was based upon anticipating the existence of a permanent atomic magnetic moment, which was incomprehensible to the classic physicists: quantum theories were to justify it but only later. Lastly, Weiss' theory on ferromagnetism, that is to say, paramagnetism with a molecular field, remained merely phenomenological, calling for both the existence of a permanent atomic moment and that of interactions of an unknown nature. But it had the great merit of introducing the new and long-disputed notion of spontaneous magnetization as well as that of an elementary domain. It is interesting to point out that it was Barkhausen's discovery of discontinuities which finally confirmed this latter notion, although it is concerned with something else.

Lastly, magnetism appeared as an isolated and autonomous field within solid-state physics, and very lightly linked to other properties.

Fifty years of interrupted progress have completely changed this outlook and have entirely integrated magnetism to the whole of solid-state physics. From now on, we have clear basic ideas about the origin of atomic moments and their various interactions. To Weiss' ferromagnetic order, characterized at low temperature by a complete and gregarious parallelism of the atomic moments, were spectacularly added the antiferromagnetic order, then the ferrimagnetic order, the helical order, and finally a very large number of other ordered struc- 
tures of varied aspects. Correlatively, the changes from one of those structures to another, in order or in complete disorder, that is to say the phase transitions, have seen their theories greatly improved. Thus the properties of ferromagnetics near to their Curie point have lost their mystery.

We could thus now hope to have a satisfactory overall view of the magnetic properties of the solid state and deem that future progress will only be made on details, as for instance on Fermi's more or less distorted surface for a given complicated material or another.

Where do we stand exactly? To answer this question truthfully, let us examine the case of iron, which is undeniably the most frequently found magnetic substance as well as the one whose technological applications are the most important.

We then have to admit that actually we can not calculate $a$ priori the value of the saturation magnetization of iron with any precision, e.g., within about 1 percent, nor the value nor even the sign of magnetic interactions, nor a fortiori their variation with temperature. Of course, we know that its magnetism comes almost exclusively from the electronic spin and that at low temperatures we are dealing with a ferromagnetic spin order, but on top of that is there an orbital antiferromagnetic order? This question remains unsolved. We could give many other examples of our uncertainties but now is not the time for such a list.

Let us now put aside the atomic scale, and broach the microscopic scale, viz. phenomena related to regions with dimensions the order of a micron. Because of the strong anisotropy of iron and of the association of Weiss' elementary domains and Bloch's walls, we can fairly well describe the mechanisms of the magnetization of a large crystal of pure iron, provided we give it an elliptical shape, rather unusual for a monocrystal, and we are not too inquisitive about what is going on at the surface, in a layer about $1-\mu \mathrm{m}$ thick.

In all the other instances, such as small monocrystals, polycrystalline iron, mixed iron and so on, we can but give a qualitative description of the phenomena. In this respect, the most striking example is that of the magnetization graph from 10$500 \mathrm{G}$ of pure polycrystalline iron, with randomly orientated crystallites. As far as I know, there is no satisfactory theory giving the value of the magnetization to within 1 percent.

The main cause for this inability lies in the powerful part played by the purely magnetic interactions and their ability to act over long distances. They cause a very complex distribution of the internal scattering fields. There does not seem to be any mathematical methods capable of dealing with these kind of problems with sufficient precision.

It should be added that the relatively satisfactory results given by the theory of the magnetization of large iron crystals arise from the high magnetic anisotropy of this metal, which causes very thin Bloch walls to appear with a high surface energy and wide elementary domains.

In a weakly disturbed crystal, the variations in magnetization in weak fields and the Rayleigh's laws that enable us to explain them originate from the movements of the walls. These are fairly well understood, but we would grasp them even better if we had a more detailed knowledge of certain properties of random functions, notably the distribution of the distances between their zero points and successive extrema.

Contrary to what many research workers think, progress in this field, and more generally speaking, in the interpretation of hysteresis, will not come from a more detailed analysis of the pinning of the walls due to a defect in such or such a crystalline network, but will come from a better mathematical knowledge and application of the interactions between the disturbances caused by a very large number of elementary defects. To sum this up; a general theory remains to be made up which would be applicable to all the possible defects, whatever their nature.

In materials that, contrary to iron, have a very weak magnetic anisotropy and thereby are intrinsically soft, such as Permalloy, the situation is far more confused. Indeed, the thickness of Bloch's walls increases indefinitely when the anisotropy tends toward zero, so that the very notion of a wall disappears. Thus, we do not have any satisfactory mathematical representation left for the magnetic structure of a Permalloy: the orientation of spontaneous magnetization continually changes in every part of it, no matter how small; there are no longer any domains, and we do not know how the magnetization variations are created. These variations have not been the object of any serious theory.

Only as a reminder, I shall mention here another topic for possible studies: the magnetic properties of very fine ferromagnetic and antiferromagnetic grains are still not very wellknown, but we must not count on rapid progress, since we are faced with uncertainties as to the stoichiometry of these grains and the difficulties in knowing the precise state of their surface.

We now come to the irreversible magnetic phenomena, that is, the ferromagnetic hysteresis and the establishment of laws that would allow a description and a forecast of the variations of the magnetization with the variations of the magnetic field within the limits of the hysteresis loop. This theoretical knowledge is all the more desirable, as permanent magnets only exist through hysteresis and form the basis for a number of varied and important applications.

At present, we only have a clear idea of the phenomena in the immediate vicinity of the origin, that is, for weak magnetizations as compared to the saturation magnetization and for weak magnetic fields as compared to the coercive field. To obtain a satisfactory representation of all the complex properties learned through experience, we would need first of all to know how to deal mathematically and completely with the simplest conceivable model-that of a set of elementary grains with rectangular hysteresis loops, randomly orientated, and coupled together by their associated magnetic dipole fields; analogous models of a few tens of coupled grains were built and showed properties which seem to be the outline of those of a real substance, and thus show that this approach would be likely to succeed.

Moreover, such theories might apply to many other irreversible nonmagnetic effects, which would be interesting. Experimental results indeed show surprising analogies between empirical laws that, for instance, describe decreasing alternate plastic deformations and magnetic hysteresis in a decreasing 
alternating field. But a close collaboration between physicists and mathematicians would be indispensible to make decisive progress in this matter. Among the wide choice of various manifestations of magnetic hysteresis, two phenomena should be mentioned: creeping motion and thermal fluctuation aftereffects.

The former, known for several hundred years, has been the source of an old way of obtaining a new magnet, called "the touch process," and consists in rubbing a steel bar several times in the same direction with a first magnet. The creeping motion consists in a gradual motion of the successive hysteresis loops that are drawn between two boundary magnetic fields, which are dissymetrical in relation to the zero field. The direction of the movement is such that the center of gravity of the cycle tends to come closer to the anhysteretic curve. After a sufficient number $n$ of loops, the motion varies as $\log n$.

A long time ago, I gave an approximate theory on this, but this phenomenon should form the subject of far more thorough theoretical investigations and of a more elaborate experimental study, which today's progress in technique should make easier.

The same conclusions apply to thermal fluctuation aftereffects. If indeed the requirements of applications in the field of high frequencies and in that of magnetic recording led to a deepening of the study of diffusion aftereffects, linked to the actual motion of ions or electrons within the crystalline network, it was not the same case for fluctuation aftereffects. The latter shows an ineluctable character and affects all ferromagnetic substances; it is proportional to the irreversible susceptibility which characterizes the magnetic state of the specimen, whereas the diffusion aftereffect is proportional to the reversible susceptibility. It is still not very well understood, notably at low temperatures. New studies would be of practical interest regarding the stability in time of permanent magnets.

The manifestations of hysteresis we have just alluded to are all related to simple variations of the magnetic field applied to the specimen and in which the same direction is usually maintained. These manifestations are already complex, but the magnetic state of a piece of iron depends on other variables than the magnetic field, for instance, on the temperature and the elastic constraints. The combined effects of simultaneous or successive variations of the temperature, of the magnetic field, and of the mechanical constraints have not been studied, or very little. This association of a scalar, a vector, and a tensor in the generation of irreversible phenomena seems likely to draw the researchers' attention and curiosity, all the more in that the little we know has shown that we deal with important effects, far beyond the limit of detection of even rudimentary devices. Moreover, important but little known applications are linked with them.

In this almost entirely unexplored field, only one area has been studied: the combined action of a magnetic field and the traction or compression on the magnetization of an iron bar. There again we deal with modern aspects of a phenomenon that has been observed for centuries: magnetization after hitting an iron bar placed in the terrestrial magnetic field. There again, it is easy to observe these effects. The alterations in the magnetization triggered off by the mere scratching of a pin on an iron bar are easily detectable with simple devices. But observing alone will not do; above all we have to explain how. Now, the theory of these effects has only been developed with success in the case of very weak constraints and very weak magnetic fields in relation to the coercive field. In all the other cases, and in particular when we cause the orientation of the specimen to vary in relation to the applied field, the observed effects-sometimes very important-remain unexplained and it is not even possible to represent them by using empirical laws.

As for the temperature, we have very few observations on its irreversible effects, such as the same decrease in the remanent magnetization, caused either by an increase or a decrease in temperature. The theory for this has barely been outlined. $A$ fortiori, it is the same thing when another variable has been superposed to the temperature.

But is it possible-and above all interesting-to develop strict theories in the instances we have just reviewed? We can indeed ponder on that, since observation frequently discloses wonderful and complicated phenomena, but above which we foresee intuitively that it would be impossible and above all vain to submit them to mathematical developments. For instance, such is the case for the splendid powder figures obtained by Bitter's method on a face of a cobalt crystal cut perpendicularly to the axis. Their ordered complexity, with a real artistic attraction, reveals the texture of superficial elementary domains which overlap in three-dimensional space. It is quite certain that for such a set of domains and walls, the search for the minimum energy configuration would discourage the best computer, no matter how powerful. In a very different field, but also infinitely simpler, the aspects of the marvelous and polymorphous snow crystals pose similar problems. Do we try to describe them by equations?

However, the problems mentioned earlier are of a different kind. We do not have to determine the state and the characteristics of each grain, each wall, and each domain constituting the elements of the medium studied, but we have to determine the characteristics and the average properties of the medium. Similarly, it is not necessary to know the individual positions and speeds of the molecules that make up a gas to determine its pressure on the walls of its container.

It is the same thing for magnetism. If, for instance, we trace a series of successive magnetization loops between two field limits, $+H_{m}$ and $-H_{m}$ symmetrical in relation to the origin, experimental results show that the macroscopic magnetization will take up the same value every time the magnetic field takes up the same value. This apparent identity certainly corresponds to distributions of elementary domains that are entirely different from one cycle to the next.

It is then a general statistical theory of the magnetic hysteresis and of a few other irreversible phenomena that we would have to develop. I felt this necessity before and tried to blaze a trail through but you would have to go farther and do so more thoroughly.

The conjuncture could be favorable, for as the program of 
this Conference shows, the work done in magnetism has never been so important. The field of magnetism appears now to be furrowed by a number of wide avenues willingly taken by a large number of research teams and which open up onto fruitful applications. But in order to know a country well, we also have to explore its paths. Since we are in a district where skiing holds a place of honor, I would dare make a comparison. With the help of ski lifts and caterpillar vehicles to flatten the tracks, the skiers can perform safely and efficiently on perfectly well-kept slopes. It is the same thing with many an explorer in magnetism, with the help of electronics, microprocessors and polarized neutrons. But let us think also of the joys of skiing out of the track despite possible avalanches. Why would some of our researchers, maybe not the fresh skiiers, not be tempted, despite the reluctance of the planners, by the satisfactions offered by exploring some lonely paths? 